\title{
Three-dimensional silicon inverse photonic quasicrystals for infrared wavelengths
}

\author{
ALEXANDRA LEDERMANN ${ }^{1,2 *}$, LUDOVICO CADEMARTIRI ${ }^{3}$, MARTIN HERMATSCHWEILER ${ }^{1,2}$, \\ COSTANZA TONINELLI ${ }^{4}$, GEOFFREY A. OZIN ${ }^{3}$, DIEDERIK S. WIERSMA ${ }^{4}$, MARTIN WEGENER ${ }^{1,2}$ \\ AND GEORG VON FREYMANN²,5
}

\author{
${ }^{1}$ Institut für Angewandte Physik, Universität Karlsruhe (TH), Wolfgang-Gaede-Straße 1, D-76131 Karlsruhe, Germany \\ ${ }^{2}$ DFG-Center for Functional Nanostructures (CFN), Universität Karlsruhe (TH), D-76131 Karlsruhe, Germany \\ ${ }^{3}$ Department of Chemistry, University of Toronto, $80 \mathrm{St}$ George Street, Toronto, Ontario M5S 3H6, Canada \\ ${ }^{4}$ European Laboratory for Nonlinear Spectroscopy (LENS) and INFM, I-50019-Sesto Fiorentino, Firenze, Italy \\ ${ }^{5}$ Institut für Nanotechnologie, Forschungszentrum Karlsruhe in der Helmholtz-Gemeinschaft, D-76021 Karlsruhe, Germany \\ *e-mail: alexandra.ledermann@physik.uni-karlsruhe.de
}

Q uasicrystals ${ }^{1-4}$ are a class of lattices characterized by a lack of translational symmetry. Nevertheless, the points of the lattice are deterministically arranged, obeying rotational symmetry. Thus, we expect properties that are different from both crystals and glasses. Indeed, naturally occurring electronic quasicrystals (for example, AlPdMn metal alloys) show peculiar electronic, vibrational and physico-chemical properties. Regarding artificial quasicrystals for electromagnetic waves, three-dimensional (3D) structures have recently been realized at $\mathrm{GHz}$ frequencies ${ }^{5}$ and $2 \mathrm{D}$ structures have been reported for the near-infrared region ${ }^{6-9}$. Here, we report on the first fabrication and characterization of 3D quasicrystals for infrared frequencies. Using direct laser writing ${ }^{10,11}$ combined with a silicon inversion procedure ${ }^{12}$, we achieve high-quality silicon inverse icosahedral structures. Both polymeric and silicon quasicrystals are characterized by means of electron microscopy and visible-light Laue diffraction. The diffraction patterns of structures with a local five-fold real-space symmetry axis reveal a ten-fold symmetry as required by theory for 3D structures.

Quasicrystals are different from both crystals and glasses: crystals have long-range translational symmetry, whereas glasses show only short-range order. Quasicrystals show long-range order but not in a repeating fashion yielding periodicity ${ }^{1-4}$ : although the local arrangements of atoms are fixed in a regular pattern, each atom has a different atom configuration surrounding it. The Laue diffraction pattern of quasicrystals can, for example, show peaks with a five- or ten-fold symmetry axis, whereas crystals can reveal only two-, three-, four- or six-fold symmetries. Quasicrystals can be viewed as a projection of a six-dimensional (6D) crystal to three dimensions ${ }^{3,4}$. Although nature provides us with $3 \mathrm{D}$ quasicrystals for electrons ${ }^{1}$, corresponding structures for light need to be fabricated artificially. Here, the projection procedure is not just a Gedanken experiment, but can rather be
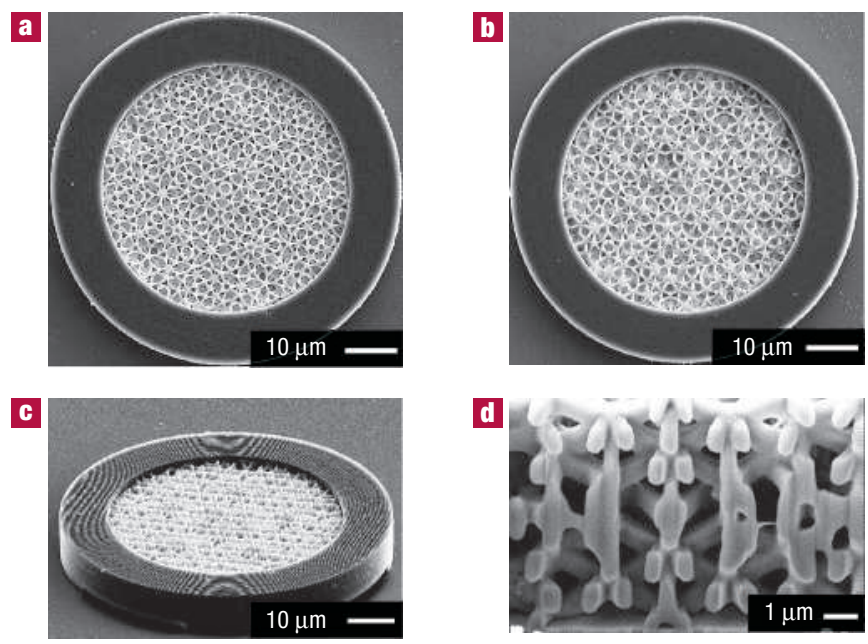

Figure 1 Electron micrographs of fabricated 3D icosahedral quasicrystal structures. a, Normal view of a sample with $I=2 \mu \mathrm{m}, N=15$ and $d=48 \mu \mathrm{m}$. The 'central atom' is in the centre of the structure. $\mathbf{b}$, The same as $\mathbf{a}$, but the 'central atom' is outside the structure. c, Oblique-incidence overview of a. d, A focused-ion-beam cut of a structure corresponding to $\mathbf{a}$, but oriented along a local two-fold symmetry axis, revealing an 3D structure.

used for the actual fabrication. This was first realized in 2005 at microwave frequencies ${ }^{5}$. The subtle but important difference between real atoms in quasicrystals and the dielectric building blocks, 'photonic atoms', in photonic quasicrystals is that real atoms can 'float' in vacuum via their binding potential. The 

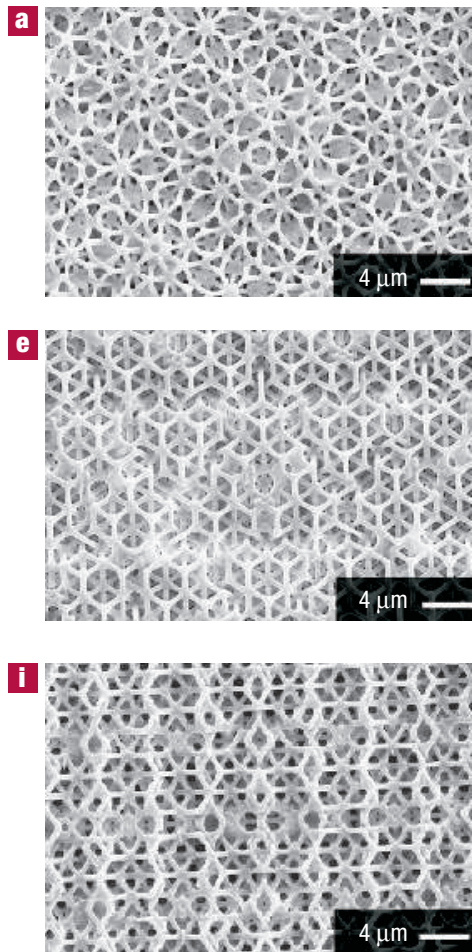

b

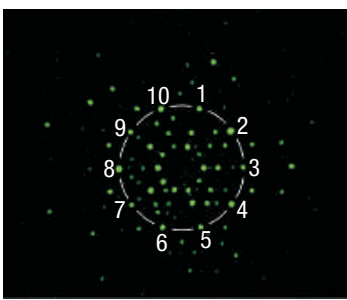

f

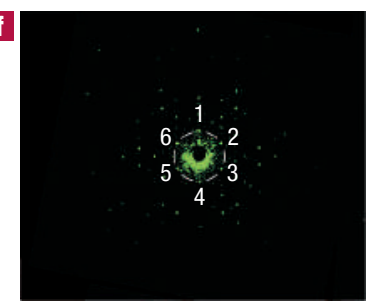

I

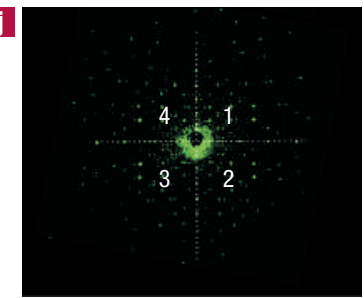

c

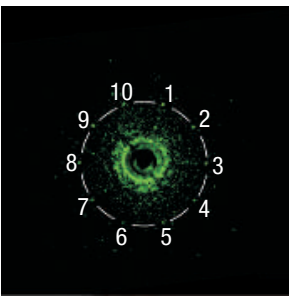

g

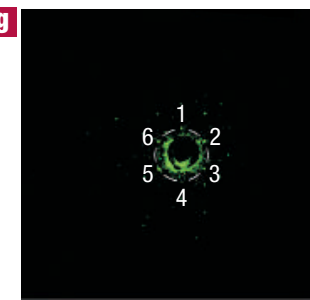

k

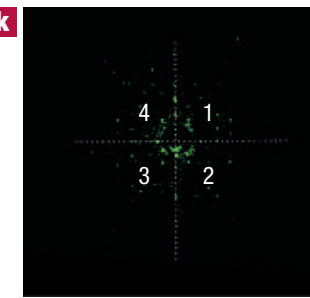

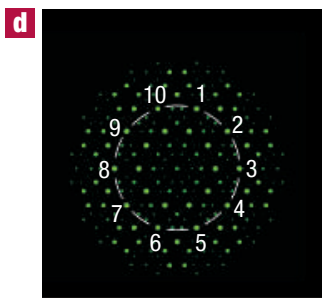

1

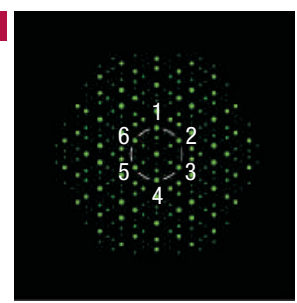

I

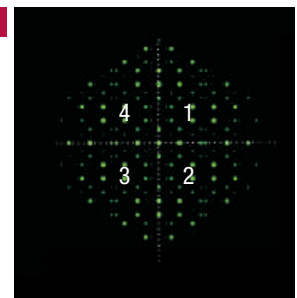

Figure 2 Laue diffraction patterns of polymeric quasicrystals. a,e,i, Close-up electron micrographs of icosahedral structures with $I=2 \mu \mathrm{m}, d=100 \mu \mathrm{m}$ and local five-fold $(N=15)$ (a), three-fold $(N=21)(\mathbf{e})$ and two-fold $(N=13)$ (i) real-space symmetry. The green spots in columns two and three are the measured Laue diffraction patterns of $3 \mathrm{D}$ quasicrystal templates using a $532 \mathrm{~nm}$ laser. $\mathbf{b}, \mathbf{f}, \mathbf{j}$, Icosahedral structures with $4 \mu \mathrm{m}$ thickness. c,g,k, Corresponding Laue diagrams for samples with $8 \mu \mathrm{m}$ thickness. d,h,l, The green spots are the calculated Laue diffraction patterns. The sizes and the brightnesses of the spots are a measure of the diffraction peak intensity.

'photonic atoms' need to be mechanically connected—unless they are held by some external force, for example, by holographic optical tweezers ${ }^{13}$. For a self-sustained permanent structure made out of a photoresist, the air voids also need to be connected, as the unexposed resist needs to be washed out during the development. Such doubly connected 3D quasicrystal structures have not been achieved at infrared frequencies so far, whereas corresponding 2D structures have revealed interesting optical properties ${ }^{6-9}$. Similarly, experiments such as anomalous diffusion of light or lasing action could conveniently be carried out on 3D quasicrystals at infrared or visible frequencies. Furthermore, doubly connected polymeric quasicrystals can be inverted or replicated by a silicon doubleinversion procedure, which was introduced recently ${ }^{12}$, or an enhanced silicon single-inversion (SSI) approach, which is new (M.H., A.L., G.A.O., M.W. and G.v.F., manuscript in preparation). The silicon replica increases the interaction of light with the structure owing to the higher index contrast, and might facilitate the observation of the above-mentioned effects.

The samples in our experiments are made by direct laser writing and subsequent SSI. The direct-laser-writing method essentially allows for the fabrication of almost arbitrarily shaped 3D photoresist structures ${ }^{10,11}$. For example, waveguides and 3D/2D/3D photonic-crystal heterostructures can also be realized ${ }^{14}$. Details of our process based on the commercial thick-film resist SU-8 can be found in ref. 11. All structures for optical diffraction experiments are surrounded by a thick solid wall, which reduces the effects of strain due to photoresist shrinkage during development. Here, we use a circular (rather than a rectangular ${ }^{11}$ ) wall in order not to break the symmetry of the structure. Furthermore, diffraction of light from the rectangular wall itself would lead to a contribution with fourfold symmetry in the Laue diffraction patterns regardless of the quasicrystal symmetry. The quasicrystal structures inside the wall are generated by the following algorithm ${ }^{4,5}$ : we start with a $6 \mathrm{D}$ simple-cubic lattice of fictitious lattice points, connected to all of their nearest neighbours by fictitious straight lines. To achieve an icosahedral quasicrystal structure, this $6 \mathrm{D}$ simple-cubic crystal is rotated around the point $(0,0,0,0,0,0)$ by the $6 \times 6$ matrix $^{15} M$ given by

$$
M=\frac{1}{\sqrt{2 \tau+4}}\left(\begin{array}{cccccc}
\tau & \tau & 0 & -1 & 0 & 1 \\
0 & 0 & 1 & \tau & 1 & \tau \\
1 & -1 & -\tau & 0 & \tau & 0 \\
\tau & -\tau & 1 & 0 & -1 & 0 \\
-1 & -1 & 0 & -\tau & 0 & \tau \\
0 & 0 & \tau & -1 & \tau & -1
\end{array}\right),
$$

where $\tau=\left(5^{1 / 2}+1\right) / 2$ is the golden mean. The second three coordinates of the resulting $6 \mathrm{D}$ vector determine whether or not a lattice point is projected. If positive, the first three coordinates represent the real-space position of this point. The projection of the 6D Wigner-Seitz cell onto 3D is shown in Supplementary Information, Fig. S1. A more detailed description of this so-called 'cut-and-project method' can be found in ref. 4 .

Pairs of connected lattice points in 6D remain connected in 3D. These fictitious connections in 3D are built up by laser-written voxels ${ }^{11}$. The 'interatomic spacing' $l$ (that is, the length of the connections) determines the order of magnitude of the photonic stop-band centre wavelength. Here, we use $l=2 \mu \mathrm{m}$ (unless stated otherwise), corresponding to infrared wavelengths. To expedite the direct-laser-writing process, each rod is written in the fast-scan 


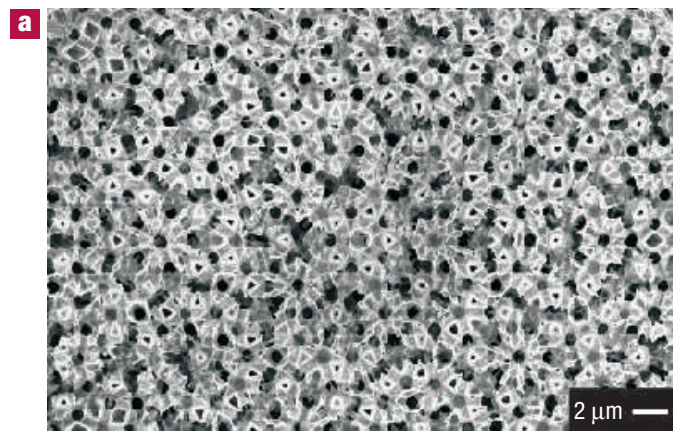

b

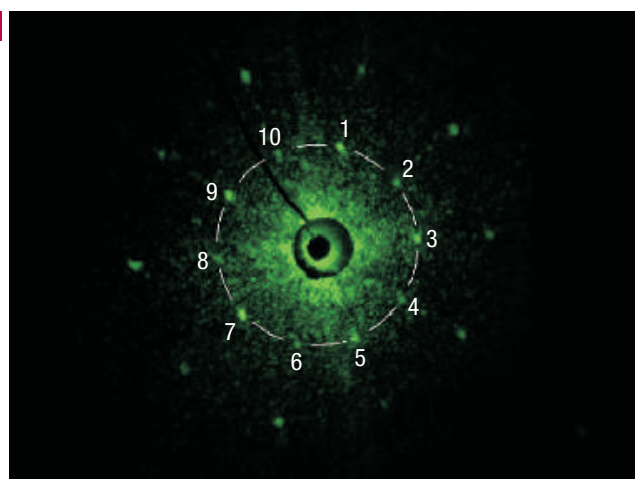

Figure 3 Silicon inverse quasicrystal. a, Electron micrograph showing the inverse of the template depicted in Fig. 2a. b, Corresponding Laue diagram (compare with Fig. 2b).

mode, that is, the piezoelectric translation stage is scanned from one end of the rod to the other end at high speed $\left(40 \mu \mathrm{m} \mathrm{s}^{-1}\right)$ while many laser pulses expose the resist. In this fashion, typical writing times for the quasicrystal structures shown below are of the order of $1.5 \mathrm{~h}$.

A gallery of electron micrographs of fabricated quasicrystal structures is depicted in Fig. 1. Figure la shows a normal view of a $d=48 \mu \mathrm{m}$ diameter structure with the above-mentioned surrounding stabilizing wall. Here, the central atom (the atom at coordinate $(0,0,0))$ with five-fold local symmetry lies in the centre of the structure. The number of lattice planes is $N=15$. In Fig. 1b, the central atom lies outside the structure, and $1 \mathrm{c}$ gives an obliqueincidence overview of 1a. Finally, Fig. 1d shows a focused-ion-beam cut of a structure similar to that in 1a, but oriented along a local two-fold axis (also see Fig. 2i), giving evidence that we have actually fabricated a $3 \mathrm{D}$ porous polymer structure.

The three rows of Fig. 2 all correspond to icosahedral quasicrystals with identical values of $l$ and $d$ but with different orientations with respect to the substrate. The first column shows electron micrographs. Figure 2a corresponds to the sample orientation of Fig. 1a, that is, to a local five-fold symmetry and $N=15$, 2e to a local three-fold $(N=21)$ and $2 \mathrm{i}$ to a two-fold $(N=13)$ symmetry axis (see Supplementary Information, Fig. S1).

The second and third columns in Fig. 2 summarize measured Laue diffraction patterns ( 4 and $8 \mu \mathrm{m}$ sample thickness for the second and third columns, respectively). In these experiments, we have focused a solid-state laser at $532 \mathrm{~nm}$ wavelength onto the samples by means of a 5 -cm-focal-length lens. The Laue diffraction pattern is scattered off a white sheet of paper and photographed (the overwhelming zeroth-order diffracted peak is blocked in order not to overload the camera). For clarity, the contrast is enhanced using image processing.

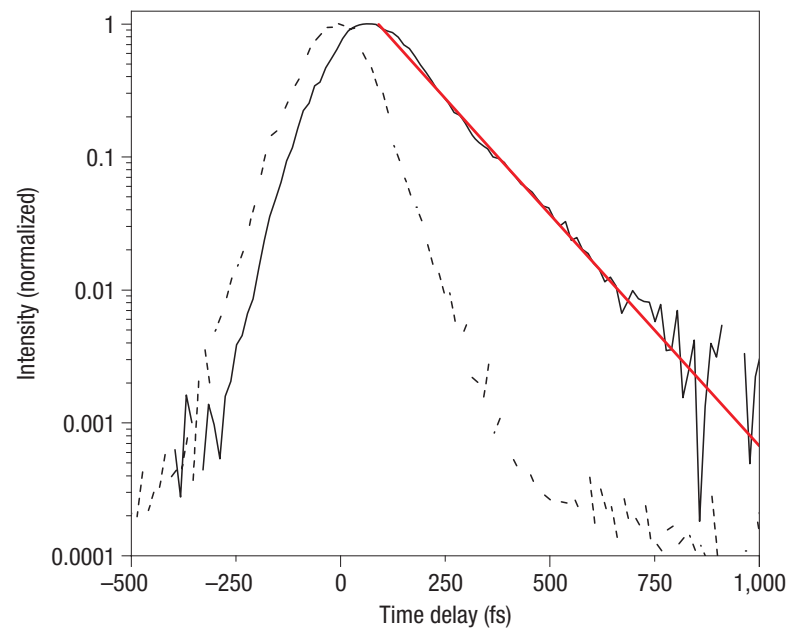

Figure 4 Diffusive light scattering of polymeric quasicrystals. Time-resolved light intensity emerging from the sample on a logarithmic scale (solid curve). The sample is a 18.7- $\mu \mathrm{m}$-thick version of the sample shown in Fig. 2e. The dashed curve is the autocorrelation of the set-up and reveals the time resolution. From the time constant of 126 fs determined from an exponential fit (red straight line) to the data, we infer a scattering mean free path of $3.4 \mu \mathrm{m}$.

The fourth column of Fig. 2 shows corresponding calculated Laue diffraction patterns. Following ref. 4, we first compute the usual diffraction pattern of the constituting $6 \mathrm{D}$ simple-cubic translational lattice. This diffraction pattern is projected onto the first three coordinates. This procedure leads to a dense set of diffraction peaks. The intensity of each peak is proportional to $\operatorname{sinc}^{2}(a)$, where $a=\pi \Delta k$, with $\Delta$ being the extension of the projected Wigner-Seitz cell and the modulus $k$ of the $3 \mathrm{D}$ vector formed by the lower three components of the $6 \mathrm{D}$ reciprocal vector. The resulting intensity of a peak is visualized in terms of both the diameter and the brightness of the corresponding green spot in Fig. 2d,h,l. Spots below a certain intensity are not shown for clarity.

Obviously, the agreement between experiment and theory is good. In particular, we obtain a ten-fold symmetry of the diffraction pattern for the five-fold axis in real space-even if the central atom lies outside the fabricated quasicrystal structure (not shown). This observation, together with the focused-ion-beam cuts discussed above, gives us confidence that we have actually succeeded in fabricating a high-quality 3D photonic quasicrystal. With increasing number of lattice planes, the diffraction peaks in Fig. 2 become sharper, as expected, at the expense of increased diffusive scattering (compare columns two and three in Fig. 2).

These high-quality SU-8 quasicrystals serve as the template in a subsequent transformation into a silicon inverse structure using SSI. We shall publish the details of the SSI process, which is a modification of the recently introduced silicon double inversion ${ }^{12}$, elsewhere (M.H., A.L., G.A.O., M.W. and G.v.F., manuscript in preparation). In brief, we coat the polymeric structures with a thin film of $\mathrm{SiO}_{2}$ in a room-temperature atomic-layer deposition process. Next, we infiltrate this $\mathrm{SU}-8 / \mathrm{SiO}_{2}$ composite with $\mathrm{Si}$, using a chemical vapour deposition process. Finally, we etch out the $\mathrm{SiO}_{2}$ using hydrofluoric acid and remove the $\mathrm{SU}-8$ via calcination. Figure 3a shows a close-up electron micrograph corresponding to the inverse of the structure in Fig. 2a, and Fig. 3b shows a corresponding measured Laue diffraction pattern, again revealing the expected ten-fold symmetry. Owing to the increased effective refractive index, the diffraction spots appear at larger angles. 
Apart from the sharp diffraction peaks, the Laue diagrams in Figs 2 and 3 also show a diffusive scattering background due to sample imperfections. To further quantify disorder, we have studied the propagation of femtosecond optical pulses through the samples (see the Methods section). The essence of this wellestablished method is that light scattered few (many) times within the sample will lead to a short (long) temporal decay. Thus, the temporal decay of the transmitted pulse is a measure of the number of scattering events. The latter is inversely proportional to the diffusion coefficient. A typical example is shown in Fig. 4 for a 18.7- $\mu \mathrm{m}$-thick SU-8 quasicrystal and a laser centre wavelength of $1.47 \mu \mathrm{m}$. The measured decay time constant of $\tau=126 \mathrm{fs}$ results in a diffusion coefficient of $D=2.6 \times 10^{2} \mathrm{~m}^{2} \mathrm{~s}^{-1}$ and a mean free path of $l_{\mathrm{s}}=3.4 \mu \mathrm{m}$. Repeating this measurement over the full series of samples and wavelengths between 1.4 and $1.6 \mu \mathrm{m}$, we obtain for $l_{\mathrm{s}}$ values in the range from 3.3 to $4.3 \mu \mathrm{m}$. These values can be compared with state-of-the-art 3D photonic crystals, which recently showed $l_{\mathrm{s}}=5-30 \mu \mathrm{m}$ (ref. 16).

Finally, we have independently measured infrared reflectance spectra in the range between 1 and $5 \mu \mathrm{m}$ wavelength (see the Methods section) for three different rod lengths $l$. We find pronounced peaks (see Supplementary Information, Fig. S2), indicating stop bands, the position of which is roughly given by twice the rod length $l$. This spectral position is consistent with down-scaling the results of ref. 5 and can also be naively expected from Bragg's law.

In conclusion, we have realized the first 3D quasicrystals for infrared frequencies. Electron microscopy, Laue diffraction, normal-incidence reflectance and light-diffusion experiments on the polymer structures reveal high sample quality. Furthermore, we have demonstrated the first conversion into a high-quality silicon inverse quasicrystal. This work paves the way for future work on low- or high-index-contrast photonic quasicrystals, for example, on anomalous diffusion of light or nonlinear optical properties, which would be difficult at microwave frequencies.

\section{METHODS}

For the time-resolved experiments, we use $150 \mathrm{fs}$ pulses tuneable in the wavelength range of 1.4-1.6 $\mu \mathrm{m}$, which we derive from an optical parametric oscillator, which we pump by an $82-\mathrm{MHz}$-repetition-rate Ti:sapphire laser oscillator. We focus these pulses onto the samples. We mix the light emerging from the sample with a reference pulse in a 1-mm-thick beta-barium-borate crystal. We record the resulting sum-frequency signal as a function of the time delay. We detect the component orthogonal to the incident linear polarization. This technique is particularly sensitive to the diffusively scattered component and is commonly applied to measure the diffusion coefficient of light transport in disordered systems. From diffusion theory we have $D=(1 / 3) v_{\mathrm{e}} l_{\mathrm{s}}$, with the energy velocity $v_{\mathrm{e}}$, which we can approximate by the phase velocity $c_{\mathrm{p}}=c_{0} / n_{\mathrm{eff}}=c_{0} / 1.3 . c_{0}$ is the vacuum speed of light and $n_{\mathrm{eff}}$ the effective refractive index of the structure. To check for consistency, the diffusion length obtained in this manner can be compared with a simple estimate on the basis of transmittance and reflectance spectra: the coherent part of the transmittance (and similarly reflectance) can be derived from the maximum of the signal versus time delay. The coherent transmittance, averaged from 1.4 to $1.6 \mu \mathrm{m}$ wavelength, is $3 \%$, the coherent reflectance $2 \%$. Thus, the worst case is that $95 \%$ of the incident light is scattered diffusively. Using Beer's law, this translates into a scattering mean free path of $l_{\mathrm{s}}=5.2 \mu \mathrm{m}$, which is comparable to $l_{\mathrm{s}}=3.3-4.3 \mu \mathrm{m}$ obtained from the temporal decay (see the main text).

Independently, we measure infrared reflectance spectra with a Fourier-transform spectrometer (Bruker Equinox 55, near-infrared halogen source) combined with an infrared microscope (Bruker Hyperion 2000, $\times 36$ Cassegrain objectives, numerical aperture $\mathrm{NA}=0.5$, liquid- $\mathrm{N}_{2}$-cooled $\mathrm{InSb}$ detector). We align the samples with their surfaces perpendicular to the optical axis. We define a circular area with $80 \mu \mathrm{m}$ diameter by an aperture in the light path of the microscope. We normalize the reflectance spectra to a silver mirror. We show three examples in Supplementary Information, Fig. S2.

Received 28 August 2006; accepted 13 0ctober 2006; published 26 November 2006.

References

1. Shechtman, D., Blech, I., Gratias, D. \& Cahn, J. W. Metallic phase with long-range orientational order and no translational symmetry. Phys. Rev. Lett. 53, 1951-1953 (1984).

2. Levine, D. et al. Elasticity and dislocations in pentagonal and icosahedral quasicrystals. Phys. Rev. Lett. 54, 1520-1523 (1985)

3. Steinhardt, P. J. \& Ostlund, S. The Physics of Quasicrystals (World Scientific, Singapore, 1987).

4. Janot, C. Quasicrystals (Clarendon, Oxford, 1992).

5. Man, W., Megens, M., Steinhardt, P. J. \& Chaikin, P. M. Experimental measurement of the photonic properties of icosahedral quasicrystals. Nature 436, 993-996 (2005).

6. Zoorob, M. E., Charlton, M. D. B., Parker, G. J., Baumberg, J. J. \& Netti, M. C. Complete photonic bandgaps in 12-fold symmetric quasicrystals. Nature 404, 740-743 (2000).

7. Kaliteevski, M. A. et al. Two-dimensional Penrose-tiled photonic quasicrystals: diffraction of light and fractal density of modes. J. Mod. Opt. 47, 1771-1778 (2000).

8. Kaliteevski, M. A. et al. Diffraction and transmission of light in low-refractive index Penrose-tiled photonic quasicrystals. J. Phys. Condens. Matter 13, 10459-10470 (2001).

9. Freedman, B. et al. Wave and defect dynamics in nonlinear photonic quasicrystals. Nature $\mathbf{4 4 0}$ $1166-1169$ (2006)

10. Kawata, S., Sun, H.-B., Tanaka, T. \& Takada, K. Finer features for functional microdevices. Nature 412, 697-698 (2001).

11. Deubel, M. et al. Direct laser writing of three-dimensional photonic-crystal templates for telecommunications. Nature Mater. 3, 444-447 (2004).

12. Tétreault, N. et al. New route to three-dimensional photonic bandgap materials: Silicon double inversion of polymer templates. Adv. Mater. 18, 457-460 (2006).

13. Roichman, Y. \& Grier, D. G. Holographic assembly of quasicrystalline photonic heterostructures. Opt. Express 13, 5434-5439 (2005).

14. Deubel, M., Wegener, M., Linden, S., von Freymann, G. \& John, S. 3D-2D-3D photonic crystal heterostructures fabricated by direct laser writing. Opt. Lett. 31, 805-807 (2006).

15. Li, F. H. \& Wang, L. C. Analytical formulation of icosahedral quasi-crystal structures. J. Phys. C: Solid State Phys. 21, 495-503 (1988).

16. Koenderink, A. F., Lagendijk, A. \& Vos, W. L. Optical extinction due to intrinsic structural variations of photonic crystals. Phys. Rev. B 72, 153102 (2005).

\section{Acknowledgements}

We acknowledge the support provided by the Deutsche Forschungsgemeinschaft (DFG) and the State of Baden-Württemberg through the DFG-Center for Functional Nanostructures (CFN) and by the European Union (EU) through contract number RII3-CT-2003-506350 (LENS) and NoE Phoremost (511616). The research of G.v.F. is further supported through a DFG Emmy-Noether fellowship. G.A.O. is Government of Canada Research Chair in Materials Chemistry. He is indebted to the Natural Sciences and Engineering Research Council of Canada for support of this research and the CFN for a Guest Professorship.

Correspondence and requests for materials should be addressed to A.L.

Supplementary Information accompanies this paper on www.nature.com/naturematerials.

\section{Competing financial interests}

The authors declare that they have no competing financial interests.

Reprints and permission information is available online at http://npg.nature.com/reprintsandpermissions/ 